\title{
An automated image analysis system for determining sea-ice concentration and cloud cover from AVHRR images of the Antarctic.
}

\begin{abstract}
.
The Australian Bureau of Meteorology operates a meteorological centre at the Casey station in East Antarctica. This centre is able to receive AVHRR data directly from the NOAA satellites and the Bureau uses these data for operational and research applications. Currently the AVHRR images are interpreted manually. However, the ICEMAPPER analysis system is able to automate this process. The system uses one set of rules to identify areas of high cloud, low cloud, open water, land ice and sea ice and another to determine sea-ice concentration. Some of the rules in ICEMAPPER were derived using information obtained from published and unpublished research, augmented by details given by practising meteorologists. Others were created by having an expert image interpreter analyse a set of representative AVHRR images and processing these analyses, using a statistical package, to produce rules which closely duplicate the manual analyses. The system was tested on six AVHRR images of the East Antarctic coastline, acquired late in the 1997/1998 summer season. It successfully identified $85 \%$ of pixels, selected from the images using a regular grid, as belonging to one of the five surface classes; high cloud, low cloud, open water, land ice or sea ice.
\end{abstract}

R.N.WILLIAMS (corresponding author)

School of Computing, University of Tasmania, PO Box 1214, Launceston

Tasmania, Australia, 7250

Phone: +61(3) 63243480

Fax: +61(3) 63243368

Email: R.Williams@utas.edu.au

K.J. MICHAEL

Institute of Antarctic and Southern Ocean Studies,

University of Tasmania,

GPO Box 252-80, Hobart, Tasmania, Australia, 7001 
and P. CROWTHER.

School of Computing, University of Tasmania,

PO Box 1214, Launceston, Tasmania, 7250

\section{Short title: Image analysis system for AVHRR images of Antarctic sea ice.}

\section{Introduction.}

The cost of carrying out scientific research in the Antarctic is high, mainly because of the difficult logistical problems that need to be overcome in order to transport and accommodate researchers in Antarctica. One of the most significant of these problems is the need to transport personnel to and from the various Antarctic stations, by ship, through potentially difficult sea-ice conditions. It is essential that ships be provided with reliable sea-ice information so they can plan their routes to and from the stations in order to transfer personnel as safely, quickly and economically as possible. The Tasmania and Antarctica Region of the Australian Bureau of Meteorology, based in Hobart, is charged with the responsibility of providing that information through the Antarctic Meteorological Centre (AMC) at the Casey station. The AMC is a joint operation of the Australian Bureau of Meteorology and the Australian Antarctic Division. Currently, Advanced Very High Resolution Radiometer (AVHRR) images from the NOAA series of satellites are received at the High Resolution Picture Transmission (HRPT) station at the AMC. Here the satellite data, of nominally $1.1 \mathrm{~km}$ horizontal resolution at the sub-satellite point and several kilometres resolution at the edge of the swath (Massom, 1991), are analysed by a meteorologist, who draws a sea-ice map, based on his or her interpretation of the images. The map is annotated with explanatory comments and transmitted to ships operating in the waters of East Antarctica (between approximately $50^{\circ} \mathrm{E}$ longitude and the International Date 
Line) but particularly in the vicinity of the three Australian stations: Casey $\left(66^{\circ} 17^{\prime} \mathrm{S}, 110^{\circ}\right.$ 32' E), Davis $\left(68^{\circ} 35^{\prime} \mathrm{S}, 77^{\circ} 58^{\prime} \mathrm{E}\right)$ and Mawson $\left(67^{\circ} 36^{\prime} \mathrm{S}, 62^{\circ} 52^{\prime} \mathrm{E}\right)$. The AVHRR data are also an essential part of the forecast service to aviation and for weather forecasting in general. Moreover, the satellite images are an important part of case studies into the evolution of weather systems and are used for climatological studies into the behaviour of polynyas (areas of relatively ice-free ocean) within the sea-ice zone off the East Antarctic coastline (Massom et al., 1998).

The aim of the ICEMAPPER project is to reduce the time required by the meteorologist to carry out this task by providing an automated system for creating the sea-ice maps under as wide a variety of different observing conditions as possible. Moreover, the use of a semiobjective tool such as ICEMAPPER is an aid to reducing the ambiguity between cloud and ice near the Antarctic coast. The ICEMAPPER system was developed using the computer programming language IDL (Interactive Data Language) because IDL provides the facilities for easily creating graphical user-interfaces and is especially suited to manipulating image and geographical data. IDL also permits development of a platform-independent system. It was also important that the system interface smoothly with the Man-computer Interactive Data Access System (McIDAS) developed by the University of Wisconsin, Madison, and currently used by the Australian Bureau of Meteorology to display and manipulate satellite data.

The ICEMAPPER system employs a graphical user-interface to handle all interaction between the user and the automated mapping process. This provides a system which is easier to use than one with a command line interface, and generally much faster to operate and less 
likely to lead to input errors. The interface has been designed to be as simple and straightforward as possible, without unnecessary complicating features.

The AVHRR images to be used by ICEMAPPER are first processed by the McIDAS meteorological package. The raw digital numbers in bands 1 and 2 (the visible bands) are calibrated to albedo values (not at this stage corrected for solar zenith angle effects) and the digital numbers in bands 3, 4 and 5 are converted to brightness temperatures. Where possible the images are referenced to known geographical locations so that at the sub-satellite point the geographical location of each point is known to an accuracy of approximately $2 \mathrm{~km}$. In cases where cloud or ice mask geographical features, errors in the location of sub-satellite pixels can be in the order of 10 to $20 \mathrm{~km}$, particularly where the orbital prediction program used to locate the radiometers in space has not had sufficient information to accurately estimate the satellite path or the pitch and roll of the spacecraft.

The calibrated image bands can then be loaded into ICEMAPPER which automatically carries out corrections to bands 1 and 2, to account for variations in solar zenith angle, and stores the result in memory for further processing.

The system can then apply a set of rules to classify each of the pixels in the image, thus creating an ice map showing regions of sea-ice, cloud, open water and continental ice depicted within the AVHRR image. This map can be displayed on the screen, saved as a disk file in GIF or postscript format, or printed out on a colour printer.

\section{Image Preprocessing.}




\subsection{Albedo and Brightness Temperature Calibrations.}

In order to perform accurate classification of the images using the rule set incorporated into the system it is necessary to have the input bands calibrated to absolute albedo values for the two visible bands and to brightness temperatures for the three infra-red bands. The ICEMAPPER system accepts images as McIDAS $\underline{\text { area files, }}$ in which the satellite data has been calibrated to albedo and brightness temperature values.

McIDAS performs albedo and brightness temperature calibrations using the calibration prefixes provided at the beginning of each scan line in the AVHRR image. These calibration procedures are outlined in (Kidwell 1991) and (NOAA 1979) but may vary between satellites depending on the perceived best practice at the time.

For the two visible bands, each digital number is converted to an albedo, using the formula: -

$$
A_{i}=S_{i} * D N_{i}+I_{i} \quad(i=1,2)
$$

where $A_{i}$ is the albedo (in \%), DN $N_{i}$ is the raw digital number, and slope $S_{i}$ and intercept $I_{i}$ are both coefficients provided within the prefix at the start of each scan line in the raw image. Although NOAA periodically publishes new coefficients obtained from checks against known ground sites, the AVHRR data used by the Bureau of Meteorology relate to the prelaunch coefficients. The Bureau is considering changing this approach. 
For the three infra-red bands, each digital number is converted to a radiance, using the formula:-

$$
R_{i}=S_{i} * D N_{i}+I_{i} \quad(i=3,4,5)
$$

where $R_{i}$ is the radiance (in $\mathrm{Wm}^{-2} \mathrm{st}^{-1} \mu \mathrm{m}^{-1}$ ), $D N_{i}$ is the raw digital number, and slope $S_{i}$ and intercept $I_{i}$ are both coefficients provided within the prefix at the start of each scan line in the raw image. These coefficients are adjusted "on the fly" by the radiometer viewing an on-board target and then sensing the infra-red emission from outer space. These two targets provide two points on a calibration curve, permitting dynamic calibration of the radiometer.

Following (NOAA 1979, Appendix A) the radiance value is then converted to an effective temperature Teff $_{i}$ (in K) using the inverse Planck Equation. The brightness temperature of a pixel $\left(\right.$ Tbrit $\left._{i}\right)$ is then assumed to vary linearly with $\operatorname{Teff}_{i}$ :-

$$
\text { Tbrit }_{i}=P_{i} * \text { Teff }_{i}+Q_{i} \quad(i=3,4,5)
$$

where $P_{i}$ and $Q_{i}$ are determined by laboratory targets. In reality the instrument has a nonlinear response, so Tbrit $_{i}$ is then used to look up a table of corrections to adjust for this nonlinearity.

\subsection{Navigation.}


As mentioned, image navigation is also carried out within McIDAS, prior to loading the image into ICEMAPPER. All images analysed in this paper were remapped from the native satellite projection to a polar stereographic projection before being imported into ICEMAPPER. As well as producing a remapped McIDAS area file, suitable for direct input into ICEMAPPER, the McIDAS navigation procedure produces a small text file containing latitudes and longitudes for each of the four corners of the remapped image. This file is loaded into ICEMAPPER with the remapped image and is used to calculate absolute geographical coordinates for all pixels in the image. These coordinates allow the two visible bands to be corrected for varying solar zenith angle (section 2.3), and they also permit a representation of the Antarctic coastline to be superimposed over the AVHRR image bands. This coastline plays a role in the rule-based classification process (section 3) and also helps the user recognise the geographical region being depicted in the AVHRR image being analysed.

The McIDAS navigation module combines basic orbital information for the NOAA satellite with ephemeris data to locate automatically a number of specific points in the image, enabling the image to be remapped to a polar stereographic projection. This navigation procedure, together with geo-referencing to known geographical features, can usually locate pixels to within a couple of kilometres at the sub-satellite point, except when cloud obscures known landmarks.

\subsection{Solar Zenith Angle Correction.}


The albedo values produced by the calibration process described in section 2.1 would be correct if the Sun were illuminating the Earth's surface from directly above. The observed albedo value can be corrected if it is divided by the cosine of $\mathrm{Z}$ (the solar zenith angle; i.e. the angle between the sun and true vertical as viewed at the point on the earth's surface whose albedo is being observed).

$$
A_{\text {correct }}=\frac{A_{\text {observed }}}{\cos (Z)}
$$

where $A_{\text {observed }}$ is the observed albedo value and $A_{\text {correct }}$ is the corrected value. This correction process assumes that the surface being illuminated is a Lambertian reflector, which is a reasonably valid assumption for sea-ice, provided that the solar zenith angle is no greater than 75 (Zibordi and Meloni 1991).

ICEMAPPER calculates the solar zenith angle from information in the NOAA image header, namely the time at which the image was produced and the geo-referencing information provided by McIDAS via the image corner coordinates file. The solar zenith angle is determined for each pixel in the image and is then used to correct the albedo values in the two visible bands, band 1 and band 2, on a pixel-by-pixel basis.

\section{Rule-base Development.}

\subsection{Rules to Distinguish Water, Ice and Cloud.}


The fundamental approach used in this system for classifying an AVHRR image is to consider the relationships between various albedo and brightness temperature values which are characteristic of the different classes to be distinguished, and then implement these relationships as a set of rules which determine an appropriate class for each pixel in the image (eg. Gesell 1989, E. Ebert Australian Bureau of Meteorology private communication 1992, Zibordi and Van Woert 1993). Some useful preliminary results were obtained using the Ebert scheme which was the simplest computationally, being specifically designed for operational classification tasks. Rule thresholds in this scheme often needed to be adjusted on an image-by-image basis, so a semi-automated system was developed in which the expert user adjusted rule thresholds where necessary to achieve acceptable performance. The rules used in this scheme could be visualised graphically as simple rectangular regions on a number of two-dimensional decision diagrams. This enabled the user to picture, from the diagrams, the changes in system behaviour which would be caused by increasing or decreasing the thresholds, making a semi-automated system feasible.

Our ultimate aim, however, was to produce a fully automated system, with no manual adjustment of rule thresholds if at all possible. In an effort to achieve this we attempted to tune the known rule-based framework to East Antarctica by producing our own set of rules in the following manner. We took a representative set of 20 images spanning the period 4 December 1996 to 5 April 1997 for areas near the Australian stations of Mawson, Davis and Casey. For each image, the following data were extracted for "selected" pixels: an assessment as to the nature of the physical entity represented by the pixel (sea ice, cloud etc.), a simulated band 1 albedo of 0 for "dark" and 1 for "light"; the band 3 and band 4 brightness temperatures; and the location of the pixel with respect to the coast. These training pixels were selected by overlaying a coarse approximately one degree latitude by four degree 
longitude grid over the image, supplemented by the four corner points of the image and by the inclusion of features (for example, rock) which, due to their limited areal extent, generally were missed by the regular grid.

Sample training data, extracted from a NOAA 14 image taken on 22 February 1997 for an area centred on $66^{\circ} \mathrm{S} 110^{\circ} \mathrm{E}$ (in the vicinity of Casey station), is given in table 1.

\section{[Insert table 1 about here]}

For each of the 344 data points extracted from the 20 images, the values of band 1 albedo ( 0 or 1); location north or south of the coast; band 3 brightness temperature; band 4 brightness temperature; and the difference between these last two parameters, were input to a statistical decision tree software package called 'S-Plus' (Clarke and Pregibon, 1992). The S-Plus program created a decision tree (Figure 1) which was able to produce the same class designation as that provided manually for $335(97.4 \%)$ of the 344 pixels for which training data were provided.

\section{[Insert figure 1 about here]}

This decision tree should be capable of providing reasonably accurate classification of previously unseen pixels in new AVHRR images, provided that these images depict regions with geographical locations within the range of locations represented by the training images (approximately $60^{\circ} \mathrm{E}$ to $120^{\circ} \mathrm{E}$ and $60^{\circ} \mathrm{S}$ to $70^{\circ} \mathrm{S}$ ) used to create the decision tree and that the times at which the new images were acquired fall within the range of acquisition times of the training images (approximately December to April). 
In order to incorporate this decision tree into the ICEMAPPER system it was converted into a rule-base consisting of 12 rules. A single rule can be derived from a decision tree by tracing through the tree, from its root, to any one of its leaves. For example, if we traced through the decision tree (figure 1) from its root down to its left-most sea-ice labelled leaf we can derive the rule:

IF brightness temperature in band $4<258 \mathrm{~K}$

AND brightness temperature in band $3<270 \mathrm{~K}$

AND coastline map indicates pixel is over the sea

AND brightness temperature in band $4>=250 \mathrm{~K}$

THEN class $=$ sea ice.

In this way, each of the 12 leaves represented on the decision tree (figure 1) has been expressed as a rule. Each of these rules has been converted into IDL code and incorporated into the ICEMAPPER system.

\subsection{Rules to see through Thin Cloud.}

In addition to the basic rules described in section 3.1 above, a number of supplementary rules, obtained from the polar remote sensing literature, have been added in order to increase the information that the ICEMAPPER system can provide. 
The system operates only on images for which the solar zenith angle is less than $75^{\circ}$ and, under these conditions, the relationship between the albedos in bands 1 and 2 can be used to discern sea-ice under thin cloud. Lee et al. (1993) have shown that the albedo of sea-ice in band 1 is significantly higher than its albedo in band 2. This difference still persists even when it is being partly obscured by a thin layer of cloud. Open water, however, has practically the same albedo in bands 1 and 2 and also thick cloud has the same albedo between these two bands. This means that if the pixel has been classified as cloud (either high cloud or low cloud) by the decision tree rule-base described in section 3.1, but the difference between the albedo in band 1 and the albedo in band 2 is greater than a specified threshold (Adiff), then the pixel is actually depicting thin cloud over ice and its classification can be changed accordingly. The following two rules achieve this :-

$$
\begin{aligned}
& \text { IF class }=\text { high cloud AND albedo1 }- \text { albedo2 }>A_{\text {diff }} \\
& \text { THEN class }=\text { thin high cloud over ice } \\
& \text { IF class = low cloud AND albedo1 }- \text { albedo2 }>A_{\text {diff }} \\
& \text { THEN class }=\text { thin low cloud over ice }
\end{aligned}
$$

Here albedo1 and albedo 2 refer to the solar zenith angle corrected albedos in bands 1 and 2 respectively and the threshold $A_{\text {diff }}$ has a specified value of $8 \%$. This value can be altered by the expert user, if required, during operational use. However, the evaluation of the ICEMAPPER system for this paper was undertaken without altering this threshold and we anticipate that satisfactory operational results will be obtainable without any need for user adjustment. 


\subsection{Rules to Estimate Sea-ice Concentrations.}

If a pixel has been classified as a sea-ice pixel, it is possible to use the albedo value of the pixel in either of the visible bands (in our case band 2) to estimate the sea-ice concentration within that pixel (Massom and Comiso 1994, Simpson and Keller 1995). An AVHRR pixel represents at least a 1.1 by $1.1 \mathrm{~km}$ area on the Earth's surface so that pixels classified as seaice will often have a mixture of open water and unresolvable solid ice floes within them. Without any sub-pixel information available, a possible method to estimate the ice concentration within the pixel is to compare the albedo in band $2\left(A_{\text {pixel }}\right)$ with the maximum albedo that would be expected for open water $\left(A_{\text {open-water }}\right)$ and the minimum that would be expected for compact ice $\left(A_{\text {compact-ice }}\right)$. We have followed the Ebert scheme in assigning an albedo of $12 \%$ as the threshold between open water and the sea-ice classes (E. Ebert, Australian Bureau of Meteorology, private communication, 1992). Then the sea-ice concentration can then be estimated using the formula (Zibordi and Van Woert, 1993) :-

$$
\text { concentration }=\frac{A_{\text {pixel }}-A_{\text {open-water }}}{A_{\text {compact-ice }}-A_{\text {open-water }}} * 100
$$

This percentage sea-ice concentration is then compared with the various sea-ice concentration categories defined by the World Meteorological Organisation (WMO 1970) and the pixel "binned" accordingly. The concentration ranges for the WMO categories are as follows: -

\begin{tabular}{|c|c|c|}
\hline very open ice & - & 1 to 3 tenths \\
\hline open ice & & 4 to 6 tenths \\
\hline close ice & - & 7 to 8 tenths \\
\hline
\end{tabular}


very close ice - 9 tenths.

\section{Performance Evaluation.}

\subsection{Performance Evaluation based on an Independent Test Data Set.}

As implied above, the internal misclassification rate of the decision tree shown in figure 1 was $2.6 \%$. In other words, $2.6 \%$ of the data used to create the decision tree shown in figure 1 were not correctly classified by the tree. The tree was tested against data which were extracted in a similar fashion to those data used to construct figure 1 but which were not used in the development of that decision tree. The test data comprised 256 data points from 9 scenes for the months of November, December, January and February, with one scene from 1991, 7 from 1992 and 1 from 1995, these years being chosen because data were readily available from them. The overall misclassification rate in the test set was $6.6 \%$. However, the performance of the system is likely to be less impressive if measures of skill are examined for individual physical features. Table 2 is a contingency table showing the performance of the decision tree shown in figure 1 against the 256 data points mentioned above.

[Insert table 2 about here]

This contingency table can be used to derive three useful measures of the "skill" of the system in classifying data points into appropriate classes. For each class, $\mathrm{X}$, three useful skill measures can be defined in the following way (Marzban 1998). Let: -

$A=$ the number of pixels of class $\mathrm{X}$ which have been identified as $\mathrm{X}$ $B=$ the number of pixels, not of class $\mathrm{X}$, which have been identified as $\mathrm{X}$ 
$C=$ the number of pixels, of class $\mathrm{X}$, which have been identified as not being $\mathrm{X}$

and $D=$ the number of pixels, not of class $\mathrm{X}$, which have been identified as not X.

Then: -

(a) the Probability of Detection $(P O D)$ may be given by $P O D=A /(A+C)$ and is a measure of the success of the system in correctly classifying pixels of class X.

(b) the False Alarm Ratio $(F A R)$ may be given by $F A R=B /(A+B)$ and measures the tendency for the system to classify pixels as being of class $\mathrm{X}$ when they are not.

and

(c) the Critical Skill Index (CSI) may be given by $C S I=A /(A+B+C)$ and is a measure of the skill of the system in correctly classifying data points of class $\mathrm{X}$ but with minimal false alarms.

It may be inferred from table 3 that while the decision tree of figure 1 shows great promise, with performance measures approaching those of a perfect system, there exists the likelihood of small but significant misclassifications in some categories. For example, the FAR for high cloud in table 3 is $17 \%$. In other words, on about $20 \%$ of occasions when the system classified the pixel as sea ice, the 'truth' was assigned as something else. On the other hand, the system appeared to correctly locate about $92 \%(P O D=0.92)$ of all sea ice identified in the test images.

[Insert table 3 about here] 


\subsection{Performance Evaluation based on Comparison with Further Manual Analyses.}

The ICEMAPPER system was tested on six previously unseen images acquired late in the 1997/98 summer season. These images were acquired between 24 February and 9 March 1998 and depicted sea ice regions off the East Antarctic coastline between $61^{\circ} \mathrm{E}$ and $124^{\circ} \mathrm{E}$ longitude. Details of the images are given in table 4.

[Insert table 4 about here]

The images were first classified manually by an expert sea-ice image interpreter (L. Cowled, Australian Bureau of Meteorology) who had had no previous involvement with the ICEMAPPER project. They were then classified automatically by ICEMAPPER and each automatic classification compared with the corresponding manual classification. Figure 2 shows the manual analysis and figure 3 the corresponding automatically-produced ice map for image CA9805701.

[Insert figure 2 about here]

[Insert figure 3 about here]

4275 individual data points were chosen from the 6 test images by selecting points on a regular grid covering each image. For each data point, the surface type classification provided by the manual analysis at that point was compared with that provided by ICEMAPPER and the results for all 4275 points have been summarised in the contingency 
table shown in table 5. If for a specific data point, the manual classification was sea ice (for example) and the ICEMAPPER classification was open water, then this point would be counted towards the number given in the open water row of the sea ice column in the contingency table (22 in this case). The performance evaluation in this section does not include a bare rock class because that class was not represented in any of the data points taken using the fixed grid approach.

[Insert table 5 about here]

[Insert table 6 about here]

Again, the performance statistics described in section 4.1 can be derived from the information in table 5 and these are shown in table 6 . There was a total of 3631 sample points, in the six images, classified correctly. This represents $85 \%$ of the 4275 tested. It may be inferred from table 6 that, while the rules incorporated into ICEMAPPER show significant promise, there exists the likelihood of significant misclassifications in some categories. For example, the false-alarm rate for high cloud was $25 \%(F A R=0.25$ in table 6$)$. In other words, on about $25 \%$ of occasions when the system classified the pixel as high cloud, the manual classification had assigned it to some other class. The relatively high $F A R$ values for high and low cloud reflect a significant degree of confusion between these two classes, which is not surprising considering the fact that a relatively arbitrary criterion is used, both by the manual interpreter and the automated system, to distinguish these two classes. On the other hand, the system appeared to correctly locate about $91 \%(P O D=0.91)$ of all sea ice identified in the test images and the false-alarm rate for this class was a relatively low $13 \%$ $(F A R=0.13)$ 
The decision tree rules being tested here, make use of brightness temperatures derived from band 3 and this band is known to suffer from significant noise problems in AVHRR sensors mounted on satellites prior to NOAA 15 . However, noise in band 3 did not noticeably affect results in this work. Most data was taken from the more recent NOAA satellites where the problem was less evident and the thresholds produced by the S-Plus package were located in such a way that any variation in channel 3 brightness temperatures resulting from the noise were insufficient to affect the classification produced by the decision tree.

The evaluation described above tested the performance of the decision tree rules. However, the manual analyses also discriminated between high cloud and high cloud over ice and between low cloud and low cloud over ice. The two 'cloud over ice' classes were used when the image interpreter was able to see through thin cloud sufficiently clearly to identify ice under it. The ICEMAPPER system is also able to see through thin cloud using some extra rules (not derived from the decision tree) which are described in section 3.2. Therefore, a separate evaluation was undertaken to determine how effectively these rules were operating. Results from this evaluation are given in table 7.

\section{[Insert table 7 about here]}

The table indicates that the system is reasonably successful in looking through the cloud, to determine whether ice is visible underneath. Of the 2216 data points which have been classified as cloud, both manually and by the ICEMAPPER system, in 1623 cases (73\%) the manual and the automated classifications agree. The system suggests that ice is visible through the cloud, where the manual interpreter sees no ice underneath, for 181 cases $(8 \%)$. This may indicate that the albedo difference threshold in this rule is set slightly too low or 
perhaps the system is able to discern the ice more easily than the manual interpreter because of its ability to closely compare the albedo levels in the two bands. The opposite situation, where the manual interpreter sees ice under cloud but where the ICEMAPPER system does not, only occurs in 16 cases (less than $1 \%$ ).

It was not possible to evaluate the rules which produced the various ice concentration estimates since these could not be estimated manually to sufficient accuracy to render any comparison meaningful. The evaluation of these concentrations will need to be carried out in future with comparison of shipboard or helicopter observations or by comparison with alternative techniques for determining sea-ice concentrations, such as passive microwave radiometers (eg. SSM/I) or synthetic aperture radar.

\subsection{Usability Evaluation in an Operational Setting.}

As with any computer system designed for operational, rather than just research use, the accuracy of the result produced is only one factor affecting the usefulness of the system in the field. It is also important that the user interface is friendly and does not impose too much extra cognitive load on the expert user, who is working under significant pressure already, particularly if working within the Antarctic environment. Therefore, an essential part of the evaluation of the system is the evaluation of its usability in an operational setting.

During the 1996/97 Antarctic summer season, the system was used at the Bureau of Meteorology Regional Office and feedback from expert users led to a number of interface improvements, including interactive contrast and brightness controls for display of all images, 
superposition of latitude and longitude grid lines on sea-ice maps and an option which allows all bands and ice-maps to be removed from the system before a new image is loaded.

The system was installed at the Antarctic Meteorological Centre (AMC), Casey, early in 1998, for evaluation purposes. It has been found to work well in differentiating sea ice, fast ice and cloud regimes. The only problems found with the system involved discrimination between thin low cloud and sea ice, with the system occasionally confusing the two. Highlevel cloud discrimination over the high Antarctic plateau also occasionally failed.

The system still has significant limitations as an operational tool, due mainly to the fact that the output needs to be produced in colour to allow adequate discrimination of the classes being represented in the image (N.D. Adams, Australian Bureau of Meteorology, private communication, 1999). The AMC-derived analyses are normally broadcast to users by high frequency facsimile transmission and the products are, by the nature of the medium, black and white, with differing sea-ice concentrations denoted by differing fill patterns.

The conclusion from the operational trial is that the ICEMAPPER system seems to perform very well and, for the novice AVHRR user, is an excellent means of learning cloud / ice discrimination using AVHRR products. For it to play a role in the operational distribution of sea-ice products at the present time, it would need to be able to produce black and white output using the fill pattern technique, currently employed in the manual analyses, to identify different classes in the image. The system is, however, ideal for providing colour sea-ice maps for those users who are able to access colour-coded images via the Internet. 


\section{Conclusions and Further Work.}

Evaluation of the ICEMAPPER system, up to this point, suggests that the system has the potential to be a useful tool for meteorologists working both at the Hobart Regional Office and at the Antarctic Meteorological Centre at Casey. Of the 4275 data points chosen within the six test images, acquired between 24 February and 9 March 1998, the system correctly identified $85 \%$, when compared with a manual interpretation of the same image by an experienced sea-ice image interpreter. The test images extend over a significant part of the East Antarctic sector, from approximately $98^{\circ} \mathrm{E}$ to $122^{\circ} \mathrm{E}$ longitude. This part of the East Antarctic was specifically chosen for the development and testing of the ICEMAPPER system because it is the region in which all three of the Australian Antarctic Stations are located. However, it is likely that the same decision tree would be applicable throughout the East Antarctic and possibly in West Antarctica as well, provided the imagery being analysed was acquired during the late summer season.

These evaluations were all done on images with solar zenith angles below $75^{\circ}$ and which were acquired over the summer period (between mid-November and mid-March). Evaluation of the performance of the system at higher solar zenith angles has indicated that the system begins to produce higher misclassification rates, probably due to the inapplicability of the Lambertian reflectance model at these higher solar zenith angles (Zibordi and Meloni 1991). We intend to investigate the use of more sophisticated reflectance models in an effort to improve the performance of the system at solar zenith angles higher than $75^{\circ}$. 
It has also been determined that the misclassification rate of the system increases when images acquired at other times of the year are classified. This would be due to changes in meteorological conditions during the year. It is to be expected that the performance of the system would deteriorate when being used for times for which the system was not trained. A new set of AVHRR images, acquired between mid-August and mid-November, are currently

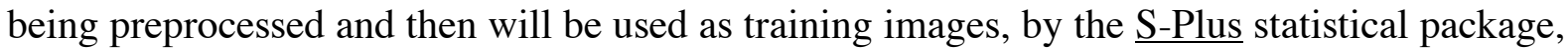
to produce a new decision tree specifically for this earlier spring period. Hopefully this tree can then be converted into a new rule-base which can be used, instead of the current one, for all future images acquired during the spring period.

\section{Acknowledgements.}

We thank staff from the Bureau of Meteorology, in Melbourne and in Hobart, who provided valuable assistance and support for this project, in particular: Dr Beth Ebert for her general advice and her initial sea-ice/cloud discrimination rules; Mr Lance Cowled who performed the manual image analyses used to evaluate the ICEMAPPER system; and Mr Doug Shepherd who processed the image data using the $\underline{\text { S-Plus }}$ software package and made suggestions for simplification of the decision tree. Other experts contributing knowledge to the rule-base were Mr Neal Young and Dr Rob Massom, from the Antarctic CRC, and we thank them for their assistance. We also thank Mr Tony Gray, from the School of Computing for his assistance in installation of the IDL remote sensing package, Dr Glenn Hyland, who provided valuable support with the IDL package and Katrina Hill for her programming 
assistance in converting the research prototype of ICEMAPPER into a useable operational tool. The research work reported in this paper was partly funded by the award of a research grant provided by the Antarctic Science Advisory Council (Project No. 2035).

\section{References.}

Clarke, L.A. and Pregibon, D.J., 1992, Tree-based Models. In Statistical Models in S. edited by J.M.Chambers and T.J.Hastie (Wadsworth and Brooks).

Gesell, G., 1989, An algorithm for snow and ice detection using AVHRR data: An extension to the APOLLO software package. International Journal of Remote Sensing, 10 (4 and 5), 897-905.

Kidwell, K., 1991, NOAA Polar Orbiting Users Guide, NOAA-NESDIS, Washington.

Lee, T.F., Atwater, S. and Samuels, C., 1993, Sea-ice-edge enhancement using polar-orbiting environmental satellite data. Weather and Forecasting, 8, 369-377.

Marzban, C., 1998, Scalar measures of performance in rare-event situations. Weather and Forecasting, 13, 753-763.

Massom, R.A., 1991, Satellite Remote Sensing of Polar Regions: Applications, Limitations and Data Availability, Belhaven Press, London. 
Massom, R. and Comiso, J., 1994, The classification of Arctic sea-ice types and the determination of surface temperature using advanced very high resolution radiometer data. Journal of Geophysical Research, 99 (C3), 5201-5218.

Massom, R., Harris, P.T., Michael, K.J. and Potter, M., 1998, The distribution and formative processes of latent-heat polynyas in East Antarctica, Annals of Glaciology, 27, 420-426.

NOAA, 1979, Technical memorandum NESS 107, W.G. Planet (ed), NOAA, Washington DC, November 1979, revised October 1988.

Simpson, J.J. and Keller, R.H., 1995, An improved fuzzy logic segmentation of sea ice, clouds, and ocean in remotely sensed Arctic imagery. Remote Sensing of Environment, 54, 290-312.

WMO, 1970, World Meteorological Organisation sea-ice nomenclature - terminology, codes and illustrated glossary, WMO/OMM/BMO-No. 259. TP. 145, 4, World Meteorological Organisation, Geneva.

Zibordi, G. and Van Woert, M.L., 1993, Antarctic sea-ice mapping using the AVHRR. Remote Sensing of Environment, 45, 155-163.

Zibordi, G. and Meloni, G.P., 1991, Classification of Antarctic surfaces using AVHRR data: A multispectral approach. Antarctic Science, 3 (3), 333-338. 


\begin{tabular}{|c|c|c|c|c|c|c|c|}
\hline $\begin{array}{l}\text { Latitude } \\
\left({ }^{\circ} \mathrm{S}\right)\end{array}$ & $\begin{array}{l}\text { Longitude } \\
\left({ }^{\circ} \mathrm{E}\right)\end{array}$ & $\begin{array}{l}\text { Physical } \\
\text { feature }\end{array}$ & $\begin{array}{l}\text { S/N of } \\
\text { Coast }\end{array}$ & $\begin{array}{l}\text { Band } 1 \\
\text { Albedo } \\
\text { (bright 1/ } \\
\text { (dark 0) }\end{array}$ & $\begin{array}{l}\text { Band } 3 \\
\text { B Temp } \\
(\mathrm{K})\end{array}$ & $\begin{array}{l}\text { Band4 } \\
\text { B Temp } \\
(\mathrm{K})\end{array}$ & $\begin{array}{c}\text { B4-B3 } \\
(\mathrm{K})\end{array}$ \\
\hline $63: 02: 30$ & $101: 53: 50$ & interference & $\mathrm{N}$ & 0 & 330.0 & 330.0 & 0.0 \\
\hline 63:02:39 & $117: 58: 46$ & high cloud & $\mathrm{N}$ & 1 & 265.0 & 247.5 & 17.5 \\
\hline $68: 19: 37$ & $119: 57: 30$ & high cloud & $\mathrm{S}$ & 1 & 283.0 & 252.5 & 30.5 \\
\hline $68: 18: 20$ & 100:00:03 & cont ice & $\mathrm{S}$ & 1 & 259.0 & 243.5 & 15.5 \\
\hline 64:00:00 & 102:00:00 & open water & $\mathrm{N}$ & 0 & 273.0 & 271.5 & 2.5 \\
\hline 65:00:00 & 106:00:00 & sea ice & $\mathrm{N}$ & 1 & 269.5 & 268.0 & 1.5 \\
\hline 66:00:00 & 110:00:00 & open water & $\mathrm{N}$ & 0 & 270.5 & 270.0 & 0.5 \\
\hline $67: 00: 00$ & 114:00:00 & high cloud & $\mathrm{S}$ & 1 & 285.0 & 256.0 & 29.0 \\
\hline 68:00:00 & 118:00:00 & cont ice & $\mathrm{S}$ & 1 & 265.5 & 257.5 & 8.0 \\
\hline
\end{tabular}

Table 1 - Typical "training data" for input to the $\underline{\text { S-Plus }}$ decision-tree package. 


\begin{tabular}{lllllllllr}
\hline & \multicolumn{7}{c}{ Classified Manually } \\
& Totals & H & L & S & W & C & R & I \\
& & & & & & & & \\
& H=high cloud & $\mathbf{4 8}$ & 6 & - & - & 4 & - & - & 58 \\
Classified & L=low cloud & - & $\mathbf{3 2}$ & 3 & - & - & - & - & 35 \\
by System & S=sea ice & - & 2 & $\mathbf{3 5}$ & - & - & - & - & 37 \\
& W=open water & - & - & - & $\mathbf{3 0}$ & - & - & - & 30 \\
& C=continental ice & - & - & - & - & $\mathbf{8 0}$ & - & - & 80 \\
& R=rock & - & - & - & 1 & - & $\mathbf{4}$ & - & 5 \\
& I= interference line & - & - & - & - & - & - & $\mathbf{1 0}$ & 10 \\
& & & & & & & & & \\
& Totals & 48 & 40 & 38 & 31 & 84 & 4 & 10 & 255 \\
\hline
\end{tabular}

Table 2 - Contingency table showing decision tree (figure 1) classification results for test data. 


\begin{tabular}{cllllllll}
\hline \multicolumn{8}{c}{ Physical Feature } \\
& & $\mathrm{H}$ & $\mathrm{L}$ & $\mathrm{S}$ & $\mathrm{W}$ & $\mathrm{C}$ & $\mathrm{R}$ & $\mathrm{I}$ \\
& & & & & & & \\
\hline & POD & 1.00 & 0.80 & 0.92 & 0.97 & 0.95 & 1.00 & 1.00 \\
Index & $F A R$ & 0.17 & 0.08 & 0.05 & 0.00 & 0.00 & 0.20 & 0.00 \\
& $C S I$ & 0.82 & 0.74 & 0.88 & 0.97 & 0.95 & 0.80 & 1.00 \\
\hline
\end{tabular}

Table 3 - Performance statistics utilising the data of table 2. 


\begin{tabular}{llll}
\hline image name & date of acquisition & region depicted & approx. image centre \\
\hline CA9805701 & 26th February 1998 & Casey Region & $65.5^{\circ} \mathrm{S}, 110.0^{\circ} \mathrm{E}$ \\
MD9806301 & 4th March 1998 & Mawson-Davis & $67.0^{\circ} \mathrm{S}, 72.0^{\circ} \mathrm{E}$ \\
CA9806201 & 3rd March 1998 & Casey Region & $65.5^{\circ} \mathrm{S}, 110.0^{\circ} \mathrm{E}$ \\
MD9806801 & 9th March 1998 & Mawson-Davis & $67.0^{\circ} \mathrm{S}, 72.0^{\circ} \mathrm{E}$ \\
SH9805501 & 24th February 1998 & Shackleton Ice Shelf & $66.0^{\circ} \mathrm{S}, 100.0^{\circ} \mathrm{E}$ \\
WS9806101 & 2nd March 1998 & West Ice Shelf & $66.5^{\circ} \mathrm{S}, 86.4^{\circ} \mathrm{E}$ \\
& & & \\
\hline
\end{tabular}

Table 4. Details of the Test Images. 


\section{Classified Manually}

$\begin{array}{llllll}\text { high } & \text { low } & \text { sea } & \text { open } & \text { cont } & \text { Totals } \\ \text { cloud } & \text { cloud } & \text { ice } & \text { water } & \text { ice } & \end{array}$

\begin{tabular}{lrrrrrr}
\hline high cloud & $\mathbf{1 1 2 0}$ & 317 & - & 1 & 46 & 1484 \\
& & & & & & \\
low cloud & 143 & $\mathbf{6 3 6}$ & 6 & 1 & 11 & 797
\end{tabular}

\begin{tabular}{|c|c|c|c|c|c|c|c|}
\hline $\begin{array}{l}\text { Classified } \\
\text { by }\end{array}$ & sea ice & 19 & 22 & 309 & 4 & 1 & 355 \\
\hline System & open water & - & 8 & 22 & 376 & 2 & 408 \\
\hline & cont ice & 36 & 3 & 2 & - & 1190 & 1231 \\
\hline & Totals & 1318 & 986 & 339 & 382 & 1250 & 4275 \\
\hline
\end{tabular}

Table 5. Contingency table comparing surface type classifications produced by ICEMAPPER with those produced by manual analyses of the six test images. 


\section{Classification}

\begin{tabular}{cllllll} 
& & $\begin{array}{l}\text { high } \\
\text { cloud }\end{array}$ & $\begin{array}{l}\text { low } \\
\text { cloud }\end{array}$ & $\begin{array}{l}\text { sea } \\
\text { ice }\end{array}$ & $\begin{array}{l}\text { open } \\
\text { water }\end{array}$ & $\begin{array}{l}\text { cont } \\
\text { ice }\end{array}$ \\
\hline \multirow{3}{*}{ Index } & $P O D$ & 0.85 & 0.65 & 0.91 & 0.98 & 0.95 \\
& $F A R$ & 0.25 & 0.20 & 0.13 & 0.08 & 0.03 \\
& $C S I$ & 0.67 & 0.55 & 0.80 & 0.91 & 0.92 \\
\hline
\end{tabular}

Table 6- Performance statistics utilising the data of table 5. 


\section{Classified Manually}

$\begin{array}{lcclc}\text { high } & \text { high cloud } & \text { low } & \text { low cloud } & \text { Totals } \\ \text { cloud } & \text { over ice } & \text { cloud } & \text { over ice } & \end{array}$

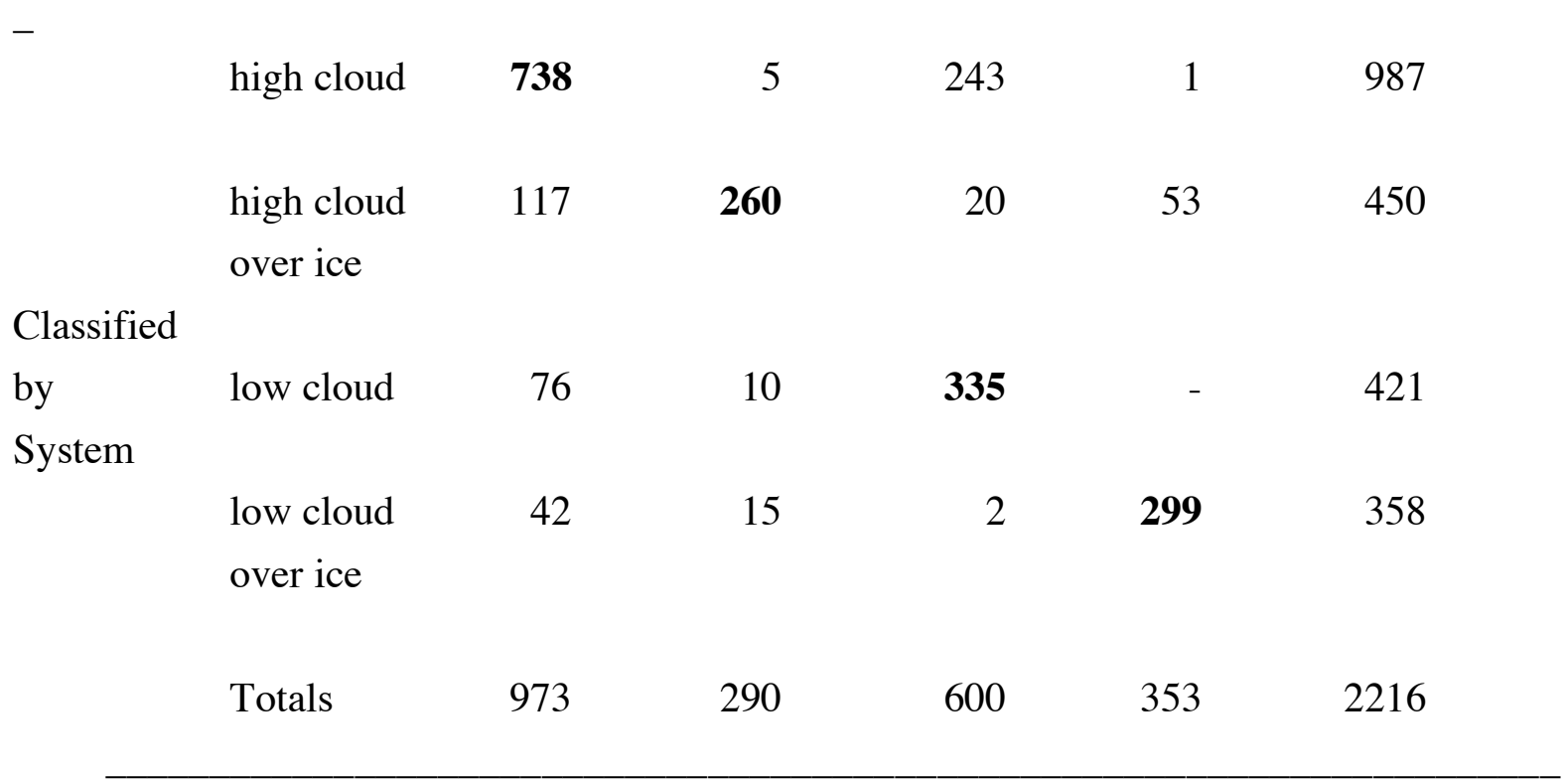

Table 7. Contingency table indicating the degree of confusion produced by ICEMAPPER between high/low cloud and high/low cloud over ice. 
Figure Captions.

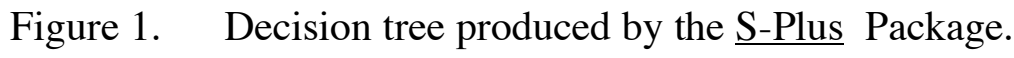

Figure 2. Band 2 of the AVHRR image CA9805701, depicting the Vincennes Bay region around Casey Station. The image was acquired on 26th February, 1998. A manual analysis (in yellow) has been superimposed on the image.

Figure 3. An automatic analysis of image CA9805701 using the ICEMAPPER system. The colours used in this map represent the following surface classes: black open water, blue - sea ice, yellow - low cloud, pink - high cloud, green - low cloud over ice, orange - high cloud over ice, red - coastline. This figure is a manual composite of two maps; the one north of the coastline being produced using rules applicable over the sea and the one south of the coastline being produced by rules applicable over the land. 